ARTICLE

\title{
Facile electron delivery from graphene template to ultrathin metal-organic layers for boosting $\mathrm{CO}_{2}$ photoreduction
}

\author{
Jia-Wei Wang (1) 1,6, Li-Zhen Qiao ${ }^{2,6}$, Hao-Dong Nie ${ }^{3}$, Hai-Hua Huang (1D 4 , Yi Li ${ }^{3}$, Shuang Yao ${ }^{2}$, Meng Liu', \\ Zhi-Ming Zhang (1) ${ }^{1 凶}$, Zhen-Hui Kang (i) ${ }^{3,5} \&$ Tong-Bu Lu ${ }^{1}$
}

Metal-organic layers with ordered structure and molecular tunability are of great potential as heterogeneous catalysts due to their readily accessible active sites. Herein, we demonstrate a facile template strategy to prepare metal-organic layers with a uniform thickness of three metal coordination layers (ca. $1.5 \mathrm{~nm}$ ) with graphene oxide as both template and electron mediator. The resulting hybrid catalyst exhibits an excellent performance for $\mathrm{CO}_{2}$ photoreduction with a total CO yield of $3133 \mathrm{mmol} \mathrm{g}^{-1} \mathrm{MOL}$ (CO selectivity of 95\%), ca. 34 times higher than that of bulky Co-based metal-organic framework. Systematic studies reveal that well-exposed active sites in metal-organic layers, and facile electron transfer between heterogeneous and homogeneous components mediated by graphene oxide, greatly contribute to its high activity. This work highlights a facile way for constructing ultrathin metal-organic layers and demonstrates charge transfer pathway between conductive template and catalyst for boosting photocatalysis.

\footnotetext{
${ }^{1}$ Institute for New Energy Materials and Low Carbon Technologies, School of Materials Science \& Engineering, Tianjin University of Technology, Tianjin 300384, China. ${ }^{2}$ School of Chemistry and Chemical Engineering, Tianjin University of Technology, Tianjin 300384, China. ${ }^{3}$ Institute of Functional Nano \& Soft Materials (FUNSOM), Soochow University, Suzhou 215123, China. ${ }^{4}$ KLGHEl of Environment and Energy Chemistry, School of Chemistry, Sun Yat-sen University, Guangzhou 510275, China. ${ }^{5}$ Institute of Advanced Materials, Northeast Normal University, Changchun 103324, China. ${ }^{6}$ These authors contributed equally: Jia-Wei Wang, Li-Zhen Qiao. ${ }^{凶}$ email: zmzhang@email.tjut.edu.cn
} 
S unlight-driven $\mathrm{CO}_{2}$ reduction has been regarded as a promising way to simultaneously achieve solar-to-chemical energy conversion and mitigate $\mathrm{CO}_{2}$ pollution ${ }^{1-3}$. Presently, solar-driven conversion of $\mathrm{CO}_{2}$-to-chemical proceeds slowly as it requires the activation of thermodynamically stable $\mathrm{CO}_{2}$ molecules and multiple electron/proton transfer processes. During the photocatalysis, various products, such as $\mathrm{CO}, \mathrm{CH}_{4}, \mathrm{HCOOH}$, and methanol, usually generate simultaneously with low selectivity, and compete with $\mathrm{H}_{2}$ evolution. To address these issues, metal-organic frameworks (MOFs), benefiting from their porous feature, ordered structure, and molecular tunability, have been widely used to mediate photocatalytic $\mathrm{CO}_{2}$ reduction ${ }^{4-18}$. In this field, Lin et al. ${ }^{16}$, incorporated $\mathrm{Re}(\mathrm{CO})_{3}(\mathrm{bpy}) \mathrm{Cl}$ (bpy = bipyridine) modules into the UiO-67 framework, achieving a total turnover number (TON) of 10.9 in photocatalytic $\mathrm{CO}_{2}$-to-CO conversion. Wang et al. achieved a high TON of 450 by using a Co-based zeolitic imidazolate framework as the cocatalyst in the presence of $\left[\mathrm{Ru}(\mathrm{bpy})_{3}\right]^{2+}$ photosensitizer $(\mathrm{PS})^{13}$. Lan et al. introduced adenine moieties into cobaltbased MOFs to drive photocatalytic $\mathrm{CO}_{2}$ reduction ${ }^{8}$. Li et al. discovered that the amine-functionalization of linking units in a series of Fe-based MOFs can improve their photocatalytic performance for $\mathrm{CO}_{2}$ reduction ${ }^{15}$. Recently, perovskite quantum dots were introduced into MOF matrices to synergistically catalyze $\mathrm{CO}_{2}$ photoreduction ${ }^{17,18}$. For $\mathrm{CO}_{2}$ reduction, great progress has been achieved with MOFs as low-cost catalysts. However, their performances were seriously hindered by the generally bulky nature with insufficient active sites and lethargic mass/charge transfer.

Nanosizing MOFs into ultrathin metal-organic layers (MOLs) can efficiently accelerate mass transport/electron transfer and create abundant readily accessible active sites to ensure high activity in various catalytic reactions ${ }^{19-34}$. Moreover, ultrathin MOFs with limited number of atoms represent ideal models to explore structure-performance relationships for further rationally constructing efficient catalysts at atomic/molecular levels. For example, a mono-carboxylic bipyridine ligand was utilized to assemble $\mathrm{Re}-/ \mathrm{Mn}-\mathrm{Ru}$ molecules into monolayer Hf-based MOLs $^{20}$, the resulting Re-Ru-based MOLs can sustainably reduce $\mathrm{CO}_{2}$ to $\mathrm{CO}$ under real sunlight for 1 week. A two-step synthesis method was used for molecular tunability to construct Ni-based MOLs, which are competent for photoreduction of diluted $\mathrm{CO}_{2}^{21}$. Up to date, a limited number of isolated MOLs can be synthesized due to their high surface energy and the serious lack of effective synthesis strategies. In principle, the MOLs can be stabilized by templates or surfactants to reduce the surface energy. However, these additional auxiliary components usually block the catalytic active sites to hinder efficient mass/charge transfer, thus severely reducing the catalytic activity. Therefore, rational design of ultrathin MOLs with functional substrates to integrate their advantages for synergistic photocatalysis will represent a promising method for constructing stable and highly efficient photocatalysts, but still a challenging task.

Herein, we demonstrate a facile template strategy to prepare MOLs with a uniform thickness of three metal coordination layers (ca. $1.5 \mathrm{~nm}$ ) by using graphene oxide (GO) as both template and electron mediator. In this composite, the conductive support not only can reduce the surface energy of the ultrathin nanosheets to isolate and stabilize the three-layer MOLs, but also can efficiently accelerate electron transfer during the $\mathrm{CO}_{2}$-to- $\mathrm{CO}$ conversion, achieving a record high $\mathrm{CO}$ yield of $3133 \mathrm{mmol} \mathrm{g}^{-1} \mathrm{MOL}$, ca. 34 times higher than that of bulky Co-MOF, much superior to those of all the state-of-the-art MOF and MOL catalysts.

\section{Results}

Synthesis and characterization. The bulky Co-MOF, [CoL $\left.\left(\mathrm{H}_{2} \mathrm{O}\right)_{2}\right] \cdot 0.5 \mathrm{H}_{2} \mathrm{O}\left(\mathrm{H}_{2} \mathrm{~L}=5\right.$ - $(1 \mathrm{H}$-1,2,4-triazol-1-yl)isophthalic acid), was synthesized via a solvothermal reaction of $\mathrm{CoCl}_{2} \cdot 6 \mathrm{H}_{2} \mathrm{O}$ and $\mathrm{H}_{2} \mathrm{~L}$ in $\mathrm{DMF} / \mathrm{H}_{2} \mathrm{O}$ at $130^{\circ} \mathrm{C}$ for $72 \mathrm{~h}$ (see the Methods for details). Single-crystal X-ray diffraction analyses reveal that Co-MOF crystallizes in a monoclinic crystal system with a space group of $C_{2 / c}$ (Supplementary Table 1). As shown in Fig. 1a, the asymmetric unit of Co-MOF contains one $\mathrm{Co}^{2+}$ cation coordinated by two aqua ligands, one $\mathrm{N}$ donor from the triazine moiety as well as three $\mathrm{O}$ atoms from two independent carboxylate groups in two $\mathrm{L}$ ligands. Through this coordination mode, one cobalt center connects with three organic ligands into a plane parallel to $b$ axis, forming a $2 \mathrm{D}$ layer-like structure. The $2 \mathrm{D}$ layers are stacked together via $\mathrm{H}$-bonding interactions $(\mathrm{O}-\mathrm{H} \cdots \mathrm{O}=2.760(4) \AA$, Supplementary Fig. 1) between aqua molecules and carboxylate oxygen atoms, showing negligible voids in the framework (Supplementary Fig. 2).

Through a lot of parallel experiments, the Cd- or Zn-based MOFs with the same ligand can be prepared (see the Methods for details). It can be noticed that Cd-MOF is isostructural to CoMOF, both exhibiting a layer-stacking structure in monoclinic crystal system with $C_{2 / c}$ space group (Supplementary Fig. 3). However, Zn-MOF displays an orthorhombic crystal system with a $P b c n$ space group. The zinc centers are in a hexa-coordinated environment completed by an aqua ligand and four L ligands into a distorted octahedral geometry, in which two equatorially coordinated organic ligands were used to link the $\mathrm{Zn}^{2+}$ ions into an uneven $1 \mathrm{D}$ chain. Furthermore, these $1 \mathrm{D}$ chains were connected into a 2D layer by the axial coordinated $\mathrm{L}$ ligand. Finally, the 2D layers are fused together by the fourth L ligand into 3D structure in Zn-MOF (Supplementary Fig. 4). The above results demonstrate the facile construction of varied MOF structures based on the L ligand with different metals.

Afterwards, the bulk phase purity of Co-MOF was confirmed by powder X-ray diffraction (PXRD; Fig. 1b) by comparison with that simulated from single crystal data, and the similar conclusion can be drawn in the cases of Cd-MOF and Zn-MOF (Supplementary Figs. 5 and 6). Importantly, the sharp peak at $26.7^{\circ}$ corresponding to $(40 \overline{4})$ face was found to represent the stacking direction of MOLs with a lattice spacing of $0.33 \mathrm{~nm}$, consistent with the distance between two 2D planes. Tightly stacking among these 2D layers in Co-MOF results in the bulky crystals with sizes over $50 \mu \mathrm{m}$, which can only be transformed into ca. $2 \mu \mathrm{m}$ crystals after ultrasonic treatment (Supplementary Fig. 7). The close stacking will inevitably decrease the exposed active sites and impede the mass transport/electron transfer during the photocatalysis.

To overcome these problems, we attempt to use $\mathrm{GO}$ as $2 \mathrm{D}$ template to graft and stabilize metal coordination layers of CoMOF to construct ultrathin MOL nanosheets. The synthetic procedure includes the incorporation of $\mathrm{Co}^{2+}$ ions into $\mathrm{GO}$ and the subsequent in situ growth of Co-MOLs with $\mathrm{H}_{2} \mathrm{~L}$ ligand on the 2D GO template (see Methods and Fig. 1c). First, both the PXRD (Fig. 1b) and FT-IR (Fig. 1d) measurements on Co-MOF, GO, and Co-MOL@GO samples reveal the effective graft of CoMOL layers on the GO support. It should be noted that the peak of $(40 \overline{4})$ face at $26.7^{\circ}$ is absent in the PXRD pattern of CoMOL@GO (Fig. 1b), indicating an obvious reduction of the stacking effect in MOLs on the GO support and the effective separation between different layers. Subsequently, the morphology of Co-MOL@GO was studied by transmission electron microscopy (TEM; Fig. 1e), where small nanoflakes $(15-20 \mathrm{~nm})$ were homogeneously distributed on the GO template. EDS mapping images indicate the even distribution of $\mathrm{Co}, \mathrm{N}, \mathrm{C}$, and $\mathrm{O}$ elements on the Co-MOL@GO sample (Supplementary Fig. 8). Atomic force microscopic results also show the distribution of nanosheets on GO substrates (Fig. 1f), with an average diameter 
a

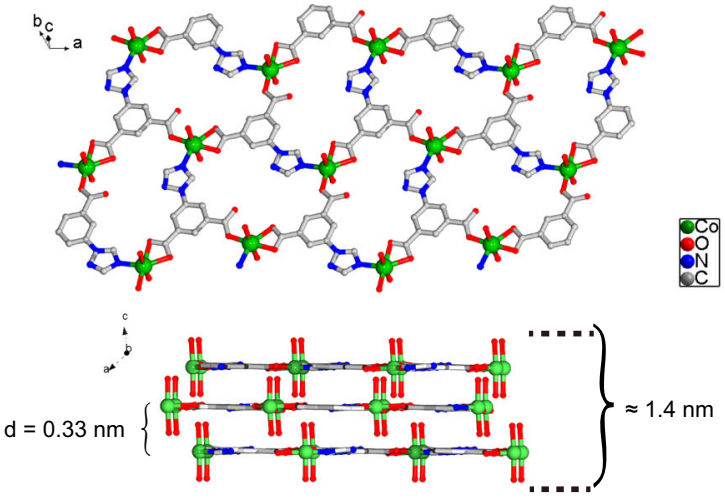

C

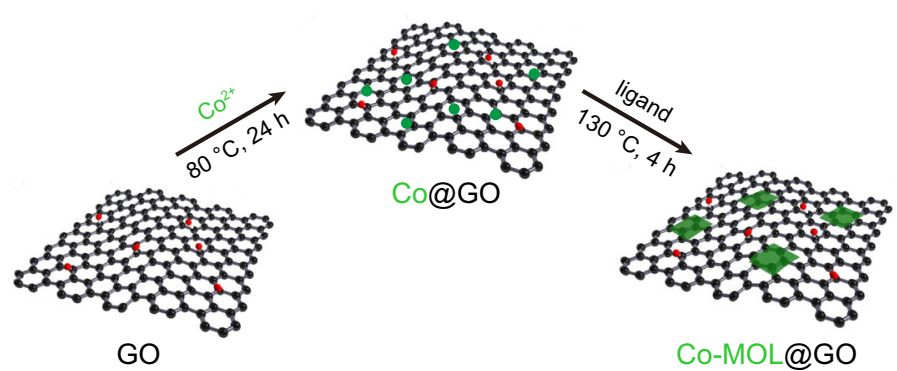

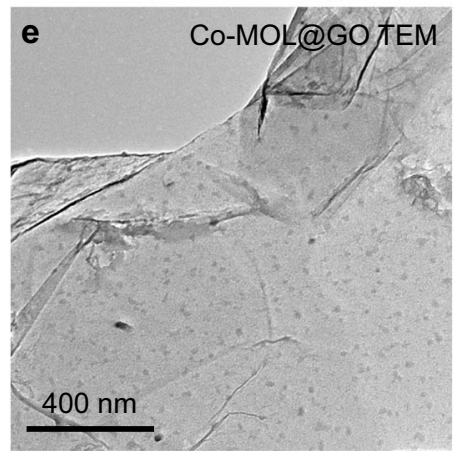

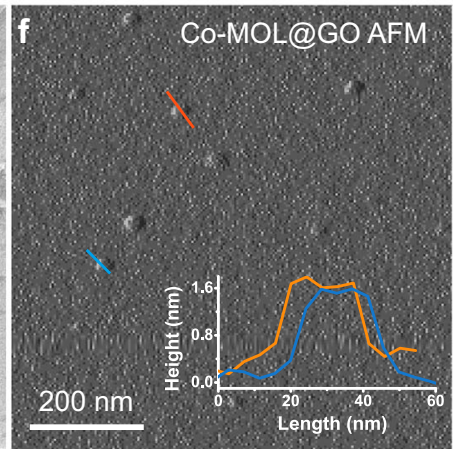

b
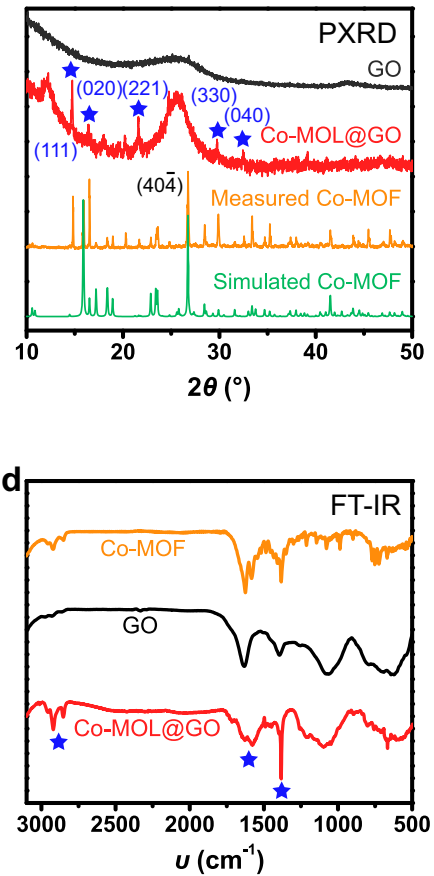

$u\left(\mathrm{~cm}^{-1}\right)$

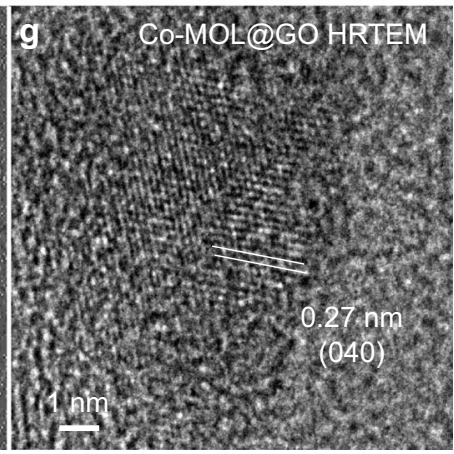

Fig. 1 Synthesis and characterization. a Layer-like structure of Co-MOF. b PXRD patterns of Co-MOF (orange), GO (black), and Co-MOL@GO (red) as well as simulated pattern of Co-MOF (green). c Synthesis of Co-MOL@GO.d FT-IR spectra of Co-MOF (orange), GO (black), and Co-MOL@GO (red). e TEM, f AFM, and $\mathbf{g}$ HRTEM results of Co-MOL@GO. The blue stars in $\mathbf{b}$ and $\mathbf{d}$ highlight the matched signals between Co-MOF and Co-MOL@GO.

of ca. $20 \mathrm{~nm}$ and a thickness of ca. $1.5 \mathrm{~nm}$, close to three metal coordination layers of $1.4 \mathrm{~nm}$ determined by the single-crystal Xray diffraction analysis (Fig. 1a). Furthermore, high-resolution TEM (Fig. 1g) measurements clearly show the crystal spacing of $0.27 \mathrm{~nm}$ in the tiny nanocrystal, in good agreement with the appearance of (040) facets, which is also consistent with the PXRD results with the characteristic peak located at $33.4^{\circ}(0.27$ $\mathrm{nm}$ ) (Fig. 1b). Accordingly, all the above experimental results can prove the successful immobilization of ultrathin Co-MOLs on the GO template, resulting in the Co-MOL@GO composite.

The morphology and composition of MOL@GO composites can be readily tuned by varying the synthetic conditions. First, we varied the loading amount of $\mathrm{Co}^{2+}$ to obtain corresponding CoMOL@GO samples that were subjected to ICP-MS (Supplementary Table 2), TEM (Supplementary Fig. 9), and PXRD measurements (Supplementary Fig. 10). These results show that different sizes and amounts of Co-MOL can be grafted on the GO substrate. Then, we also tried to produce MOL@GO hybrids by loading $\mathrm{Cd}^{-}$and $\mathrm{Zn}$-based MOFs on the GO substrate. Interestingly, Cd-MOL@GO can be prepared by a series of parallel experiments, as determined by corresponding PXRD and TEM results (Supplementary Figs. 5 and 11). We have adjusted a variety of experimental conditions to realize the loading of
Zn-MOF on the GO substrate; however, no nanosheets can be observed on the GO support, and the envisioned "Zn-MOF@GO" sample only showed indiscernible signals (Supplementary Fig. 6). By a detail analysis, it can be concluded that successful immobilization of ultrathin MOLs on GO depends on the crystallographic structure, in which the flat layered structures of Co/Cd-MOF should be more suitable for the co-plane $\pi-\pi$ interaction with GO to build the 2D-2D MOL@GO composites. Overall, these results further confirm that the GO template synthesis represents a facile strategy for the synthesis of ultrathin MOL nanosheets.

With the verified morphology of Co-MOL@GO, a series of additional measurements were operated to verify the changes of Co-MOLs and GO in Co-MOL@GO sample. Initially, X-ray photoelectron spectroscopy (XPS; Fig. 2a) reveals a negative shift in the binding energies of Co 2p in Co-MOL@GO compared to those of Co-MOF. This observation indicates the existence of interactions between Co-MOF and GO surface, which can facilitate the charge transfer between $\mathrm{Co}-\mathrm{MOF}$ and $\mathrm{GO}$ to impede the recombination of charge carriers ${ }^{35}$. Moreover, GO substrate was substantially reduced under the solvothermal condition, which was confirmed by the Raman spectra of CoMOL@GO with an increased ratio between defective bands $\left(I_{\mathrm{D}}\right)$ 

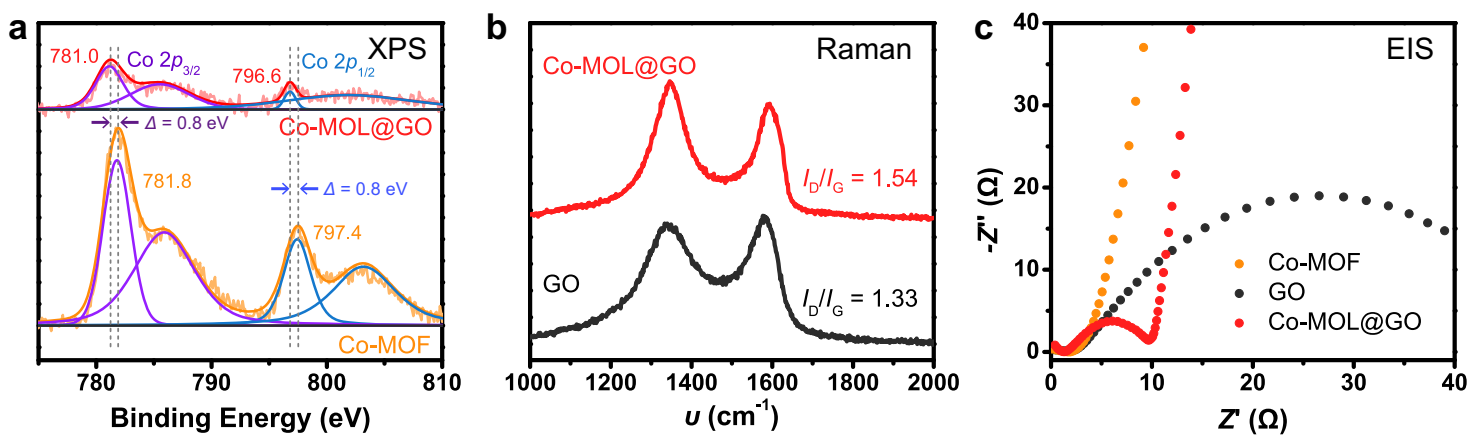

Fig. 2 Comparison between Co-MOL@GO and Co-MOF. a Co 2p XPS spectra of Co-MOL@GO (red) and Co-MOF (orange).b Raman spectra of Co-MOL@GO (red) and GO (black).c EIS spectra of Co-MOL@GO (red), GO (black), and Co-MOF (orange).

and graphitic band $\left(I_{\mathrm{G}}\right)$ relative to that of GO (1.54 vs. 1.33$)$ (Fig. $2 \mathrm{~b})^{36}$. The reduced GO can be more conductive and feasible for electron transfer during photocatalytic process. This conclusion was further supported by the results of electrochemical impedance spectroscopy (EIS), where Co-MOL@GO exhibits a smaller charge-transfer resistance compared to those of Co-MOF and GO (Fig. 2c). Overall, all above results suggest that CoMOL@GO can be a good candidate as a catalyst for photocatalytic $\mathrm{CO}_{2}$ reduction with its advantages in abundantly exposed active sites, excellent mass transport, and chargetransfer ability.

Photocatalytic $\mathrm{CO}_{2}$ reduction. The catalytic performance of CoMOL@GO for visible-light-driven $(\lambda=450 \mathrm{~nm}) \mathrm{CO}_{2}$ reduction was investigated in a $\mathrm{CO}_{2}$-saturated $\mathrm{CH}_{3} \mathrm{CN} / \mathrm{H}_{2} \mathrm{O}(v: v=4: 1)$ solution. $\mathrm{Ru}(\mathrm{phen})_{3}\left(\mathrm{PF}_{6}\right)_{3}$ (phen $=1,10$-phenanthroline; denoted as RuPS) was employed as the PS. Co-MOF, Co@GO, and GO were used as the catalysts in the control experiments. The gaseous products, $\mathrm{CO}$ and $\mathrm{H}_{2}$, were analyzed by gas chromatography, and the liquid products, i.e., $\mathrm{HCOOH}$, were checked by ion chromatography. As shown in Fig. 3 and Table 1, the main products are $\mathrm{CO}$ and $\mathrm{H}_{2}$, and no liquid products can be detected. Remarkably, a CO yield of $216.2 \mathrm{mmol} \mathrm{g}^{-1}$ with a high selectivity of 95\% can be achieved during $12 \mathrm{~h}$ irradiation of Co-MOL@GObased photocatalytic system, after optimization by varying the loading amount of Co-MOL (Supplementary Table 2 and Supplementary Fig. 12). In comparison, the photocatalytic experiment with bulky Co-MOF as the catalyst affords a $\mathrm{CO}$ yield of $91.5 \mathrm{mmol} \mathrm{g}_{\mathrm{MOF}^{-1}}$, much smaller than that of Co-MOL@GOcontaining system, as well as a lower selectivity of $82 \%$. Control experiments reveal that $\mathrm{GO}$ shows no activity toward $\mathrm{CO}_{2}$ reduction under this photocatalytic condition, indicating that the MOLs should be the real active species in the Co-MOL@GO sample. As a result, the $\mathrm{CO}$ yield can be estimated as $3133 \mathrm{mmol}$ $\mathrm{g}_{\mathrm{MOL}}{ }^{-1}$ with the TON of $1065 \mathrm{ca} 34$ times higher than that of bulky Co-MOF under similar condition, indicative of the remarkable intrinsic catalytic activity of ultrathin Co-MOF in CoMOL@GO. Impressively, in terms of CO yield and selectivity, the

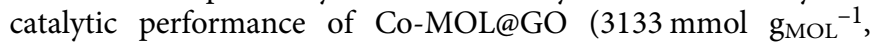
95\%) is comparable to most state-of-the-art MOF catalysts for visible-light-driven $\mathrm{CO}_{2}$ reduction, such as $2 \mathrm{D}-\mathrm{Ni}_{2} \mathrm{TCPE}^{37}$ (20 mmol g-1, 97\%, TON 13.9), Ni MOLs ${ }^{21}\left(25 \mathrm{mmol} \mathrm{g}^{-1}, 98 \%\right.$, TON 8), MAF-X27l-OH ${ }^{10}\left(25.4 \mathrm{mmol} \mathrm{g}^{-1}, 98 \%\right.$, TON 2124$)$, Co-ZIF-913 $\left(209 \mathrm{mmol} \mathrm{g}^{-1}, 58 \% \text {, TON 89.6), Ni(TPA/TEG }\right)^{38}$ $\left(47 \mathrm{mmol} \mathrm{g}^{-1}, 99 \%\right.$, TON 11.5), and other examples listed in Supplementary Table 3 . We further scaled up the reactor by five times to minimize the measurement error and get closer to realistic applications (see Methods and Supplementary Fig. 13 for details), which also afforded good CO yield (3467 $\mathrm{mmol} \mathrm{g}_{\mathrm{MOL}^{-1}}{ }^{-1}$ and selectivity (94\%) within $10 \mathrm{~h}$ of irradiation. In addition, the photocatalytic system with Co@GO as the catalyst could also produce $\mathrm{CO}$ and $\mathrm{H}_{2}$ under the same conditions, but with a much smaller amount than that of Co-MOL@GO (Supplementary Fig. 14), showing that the formation of Co-MOL nanosheets on $\mathrm{GO}$ is the key to achieve high-performance $\mathrm{CO}_{2}$ reduction. Accordingly, GO template strategy is promising to fabricate highperformance catalysts for photocatalytic $\mathrm{CO}_{2}$ reduction. These comparative results clearly demonstrate the much-enhanced intrinsic catalytic activity of 2D-nanosized Co-MOL in contrast to the bulky Co-MOF and other samples. This enhancement can be mainly attributed to its great exposure of catalytic active sites enabled by the ultrathin feature of the MOL, and the incorporation of graphene as charge-transfer mediator.

Thermal and chemical stability of Co-MOF and Co-MOL@GO were carefully investigated. For Co-MOF, thermogravimetric analysis (TGA) was conducted to investigate its thermal stability. As shown in Supplementary Fig. 15, the TGA curve shows three continuous weight losses from 95 to $320^{\circ} \mathrm{C}$, suggesting the loss of lattice and coordinated water molecules in the cavity of bulky CoMOF. It could also be observed that a thermal decomposition of Co-MOF took place until heating up to $400^{\circ} \mathrm{C}$, revealing its high thermal stability. Meanwhile, the bulk Co-MOF was soaked in a $\mathrm{CO}_{2}$-saturated $\mathrm{CH}_{3} \mathrm{CN} / \mathrm{H}_{2} \mathrm{O}(v: v=4: 1)$ solution containing $0.3 \mathrm{M}$ TEOA, a reaction medium for photocatalytic $\mathrm{CO}_{2}$ reduction. After 1 day, the solid samples were isolated for subsequent PXRD measurements. No obvious difference of the PXRD pattern can be observed compared to that of as-synthesized sample (Supplementary Fig. 16). These results demonstrate remarkable thermal and chemical stability of this Co-MOF, assuring its robustness in photocatalytic $\mathrm{CO}_{2}$ reduction. For Co-MOL@GO, PXRD pattern of the solid sample isolated from the photocatalytic system shows similar signals with that of as-prepared Co-MOL@GO, indicating the intact crystalline composition of Co-MOL@GO catalyst after photocatalysis (Supplementary Fig. 17). Moreover, recycle experiments showed no substantial decrease in the activity after three runs of photocatalytic reactions, confirming the retained activity of the Co-MOL@GO catalyst (Fig. 3b). On the other hand, isotope labeling experiment with ${ }^{13} \mathrm{CO}_{2}$ shows that ${ }^{13} \mathrm{CO}$ is the main product in this photocatalytic system (Fig. 3c), manifesting that the $\mathrm{CO}$ product really derives from $\mathrm{CO}_{2}$ rather than the decomposition of TEOA, RuPS, GO, or Co-MOLs. All these results demonstrate the excellent stability of Co-MOL@GO in the photocatalytic $\mathrm{CO}_{2}$-to- $\mathrm{CO}$ conversion.

Electron transfer pathway. To elucidate the electron transfer pathway, the emission quenching experiments of RuPS were conducted in detail with the quenchers including Co-MOL@GO, Co-MOF, and TEOA (Fig. 3d, e and Supplementary Fig. 18). In the fluorescence spectra of RuPS, an emission peak at $598 \mathrm{~nm}$ was detected with the excitation at $450 \mathrm{~nm}$. Upon the gradual addition 


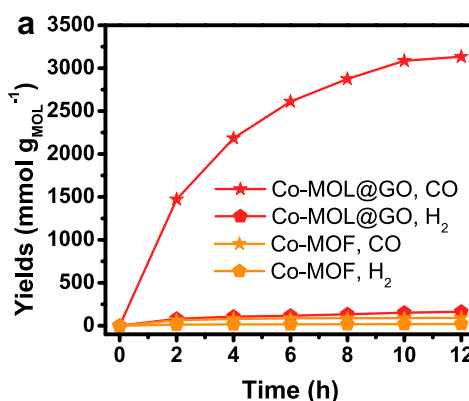

d

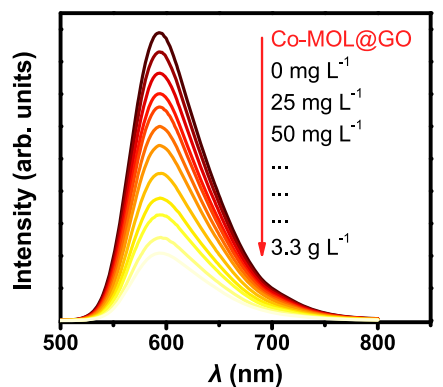

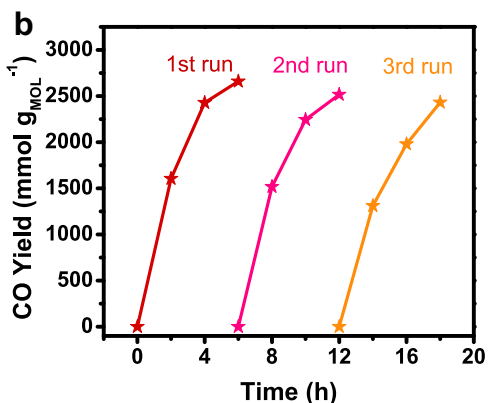

$\mathbf{e}$

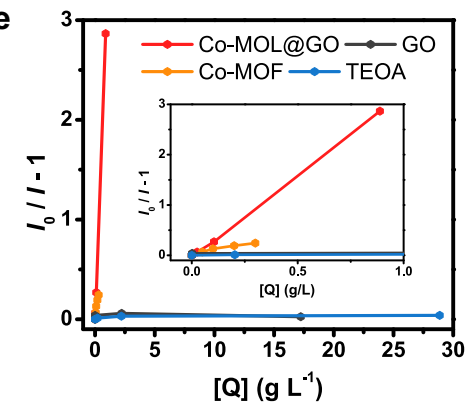

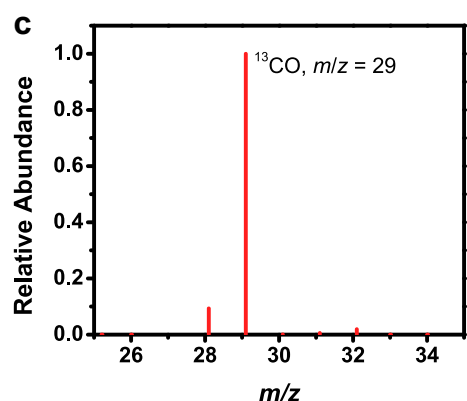

$\mathbf{f}$

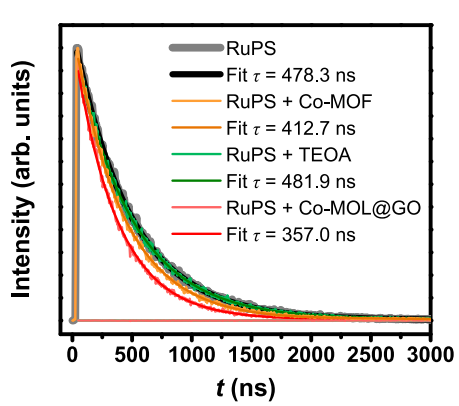

Fig. 3 Photocatalytic $\mathbf{C O}_{2}$ reduction. a Time profiles of $\mathrm{CO}$ (star) and $\mathrm{H}_{2}$ evolution (pentagon) catalyzed by 10 mg L-1 $\mathrm{Co}-\mathrm{MOL} @ \mathrm{GO}$ (red) and Co-MOF (orange) with the irradiation of a blue LED $(450 \mathrm{~nm})$ in $\mathrm{CH}_{3} \mathrm{CN} / \mathrm{H}_{2} \mathrm{O}(\mathrm{v}: \mathrm{V}=4: 1)$ solution under $1 \mathrm{~atm} \mathrm{CO}_{2}$. b Recycle experiments of $\mathrm{CO}$ production with Co-MOL@GO as the catalyst. c ${ }^{13} \mathrm{CO}_{2}$ isotope labeling experiments. $\mathbf{d}$ Fluorescence spectra of a $\mathrm{CH}_{3} \mathrm{CN} / \mathrm{H}_{2} \mathrm{O}(\mathrm{v}: \mathrm{v}=4: 1)$ solution containing 0.4 mM RuPS in the presence of 0 3.3 $\mathrm{g} \mathrm{L}^{-1}$ of Co-MOL@GO with the excitation at $450 \mathrm{~nm}$. e Stern-Volmer plot of fluorescence by the quenchers of Co-MOL@GO (red), GO (black), Co-MOF (orange), and triethanolamine (TEOA; blue) vs. the mass concentrations ([Q]). f Time-resolved absorption spectra of 0.4 mM RuPS

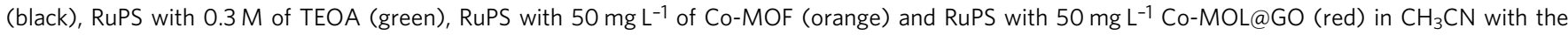
excitation wavelength of $450 \mathrm{~nm}$.

Table 1 Photocatalytic results for $\mathrm{CO}_{2}$ reduction to $\mathrm{CO}^{\mathrm{a}}$.

\begin{tabular}{|c|c|c|c|c|}
\hline Entry & Catalyst & $\begin{array}{l}\text { CO yield } \\
\left(\mathrm{mmol} \mathrm{g}^{-1}\right)\end{array}$ & $\begin{array}{l}\mathrm{H}_{2} \text { yield } \\
\left(\mathrm{mmol} \mathrm{g}^{-1}\right)\end{array}$ & CO (\%) \\
\hline 1 & Co-MOL@GO & $216.2(3133)^{b}$ & $11.2(162)^{b}$ & 95 \\
\hline 2 & Co-MOF & 91.5 & 19.8 & 82 \\
\hline 3 & Co@GO & 66.7 & 3.16 & 95 \\
\hline 4 & $\mathrm{GO}$ & N.D. & $<0.10^{c}$ & N.A. \\
\hline 5 & No catalyst & N.D. & $<0.10^{c}$ & N.A. \\
\hline
\end{tabular}

of Co-MOL@GO, the efficiency of fluorescence quenching was far higher than those obtained by addition of bulky Co-MOF and $\mathrm{GO}$, indicating that the ultrathin feature of MOL and the incorporation of graphene mediator are beneficial to electron transfer. The $K_{\mathrm{sv}}$ quenching constants of RuPS quenched by CoMOL@GO was calculated as $3250 \mathrm{Lg}^{-1}$ by Stern-Volmer $\operatorname{plot}^{39,40}$, much higher than that obtained by addition of Co-MOF $\left(813 \mathrm{~L} \mathrm{~g}^{-1}\right)$, and no obvious quenching can be observed in the presence of isolated GO. These results suggest the fast electron transfer between homogeneous PS* and heterogeneous CoMOL@GO catalyst mediated by GO, affording the high catalytic performance ${ }^{39,40}$. Furthermore, control experiments show that negligible fluorescent quenching can be detected by addition of various amounts of TEOA (Fig. 3e), indicating that the exited RuPS* was directly quenched by Co-MOL@GO via an oxidation quenching mechanism.

The acceleration of charge transfer was confirmed by timeresolved absorption spectroscopy. As shown in Fig. 3f, the kinetic traces for excited RuPS show that the lifetime of RuPS* (478.3 ns) was much longer than that obtained in the presence of Co-MOF ( $412.7 \mathrm{~ns}$ ) catalyst, and this lifetime can be further shortened to be 357.0 ns when Co-MOL@GO composite was present. These results suggest the rapid separation and migration of photogenerated charge carriers between homogeneous PS and heterogeneous catalyst with graphene as the mediator. In the presence of TEOA, the excited lifetime of RuPS* was similar to that of isolated RuPS (481.9 vs. $478.3 \mathrm{~ns}$ ), further confirming the oxidative quenching electron transfer pathway in this photocatalytic reaction for $\mathrm{CO}_{2}$ reduction ${ }^{41}$.

Furthermore, the acceleration of electron transfer mediated by $\mathrm{GO}$ and the roles of different components in the photocatalytic system were evaluated by in situ transient photovoltage (TPV) measurements on GO, Co-MOF, RuPS, and Co-MOL@GO. As shown in Fig. 4a, the photocurrent response of GO is the highest among the detected samples, and its curve in the $\mathrm{CH}_{3} \mathrm{CN} / \mathrm{H}_{2} \mathrm{O}$ $(v: v=4: 1)$ medium is similar to that in the air (Supplementary Fig. 19). Figure $4 \mathrm{~b}$ shows that the photocurrent intensity of CoMOF/RuPS mixture is higher than that of isolated components of Co-MOF and RuPS. These results suggest a coupling effect between Co-MOF and RuPS that can enhance the signal, while the response of GO is still stronger than that of Co-MOF/RuPS (Fig. 4c). Then, the photocurrent intensity decreased when GO was mixed with RuPS or Co-MOF (Co-MOL), respectively. Especially, the photocurrent intensity of Co-MOL@GO composite is much lower than that from the combination of GO and RuPS (Fig. 4d). These observations infer the electron transfer pathway, in which the electrons can be transferred to both CoMOL and RuPS from the GO surface, and Co-MOL can accept electrons more easily than RuPS.

To determine the active centers, the TPV measurements were performed in different atmospheres of $\mathrm{N}_{2}$ and $\mathrm{CO}_{2}$ to evaluate the real catalytic active centers (Fig. 4e-h). As shown in Fig. 4e, f, similar photocurrent intensities were observed in the curves of either GO or RuPS under $\mathrm{N}_{2}$ or $\mathrm{CO}_{2}$. In contrast, a sharp decrease 

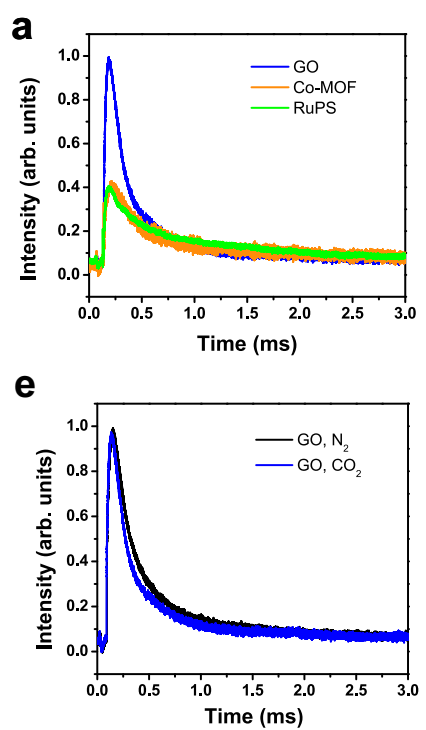

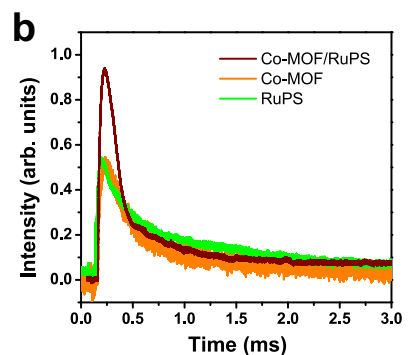

f

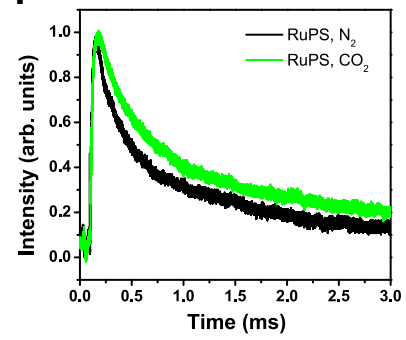

C

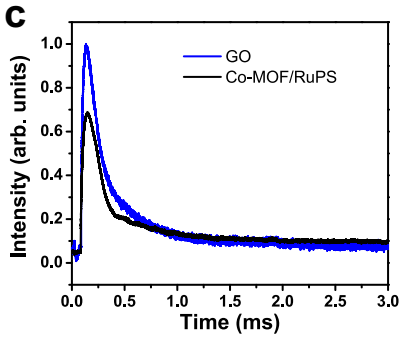

g

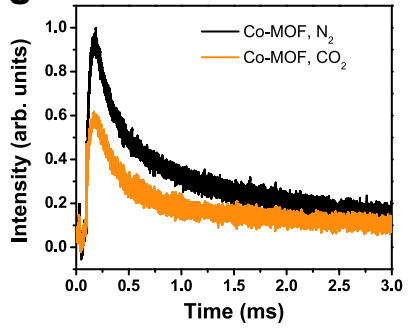

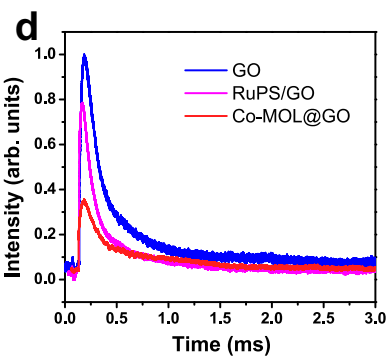

h

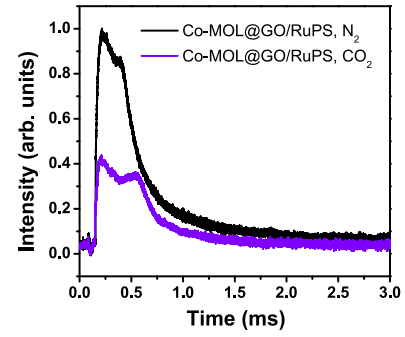

Fig. 4 In situ transient photovoltage. Comparison of the in situ TPV curves a among GO (blue), Co-MOF (orange), and RuPS (green) under $\mathrm{N}_{2} ; \mathbf{b}$ among Co-MOF/RuPS mixture (crimson), Co-MOF (orange), and RuPS (green) under $\mathrm{N}_{2}$; c between GO (blue) and Co-MOF/RuPS mixture (black) under $\mathrm{N}_{2}$; d among GO (blue), RuPS/GO mixture (pink), and Co-MOL@GO (red) under $\mathrm{N}_{2}$. Comparison of the in situ TPV curves under $\mathrm{N}_{2}$ (black) or $\mathrm{CO}_{2}$ (respective color) with e GO (blue), f RuPS (green), g Co-MOF (orange), or h Co-MOL@GO/RuPS mixture (violet), respectively.

of photocurrent intensity was detected with Co-MOF under $\mathrm{CO}_{2}$ in comparison with that under $\mathrm{N}_{2}$ (Fig. $4 \mathrm{~g}$ ). These results reveal that the photocatalytic $\mathrm{CO}_{2}$ reduction should occur on the surface of Co-MOF. That is, Co-MOL represents the active component in the system. To further confirm this proposal, the TPV of CoMOL@GO/RuPS mixture was performed, and a similar trend to that of Co-MOF was observed, confirming the role of Co-MOL as the active center (Fig. 4h). Overall, the above TPV analyses confirm that Co-MOL is the active component of photocatalytic $\mathrm{CO}_{2}$ reduction and that $\mathrm{GO}$ serves as the electron mediator to deliver electrons to Co-MOL.

Mechanistic studies. As the Co-MOL is the active component in photocatalysis, its molecular catalytic mechanism was further investigated by the electrochemical measurements. First, we studied the electrochemical behavior of GO and Co-MOL@GO loaded on the surface of the glassy carbon electrode, respectively. As shown in Supplementary Fig. 20a, the irreversible waves at ca. $-0.75 \mathrm{~V}$ versus normal hydrogen electrode (vs. NHE) were both observed in the cyclic voltammograms (CVs) of Co-MOL@GO and GO under $\mathrm{N}_{2}$, where the reduction currents are mainly attributed to the reduction events at GO. To avoid the interference of GO, Co-MOF was directly employed to investigate the redox behavior of the MOF catalyst. As shown in Supplementary Fig. 20b, a quasi-reversible redox couple at $E_{1 / 2}=-0.76 \mathrm{~V}$ vs. NHE (reduction wave at $-0.85 \mathrm{~V}$ ) appeared in the $\mathrm{CV}$ of Co-MOF under $\mathrm{N}_{2}$, corresponding to $\mathrm{Co}{ }^{\mathrm{II} / \mathrm{I}}$ reduction. Upon purging $\mathrm{CO}_{2}$ into the system, an irreversible reduction wave peaking at $-0.94 \mathrm{~V}$ vs. NHE with a relatively large current was detected, indicating a chemical step driven by $\mathrm{Co}^{\mathrm{II} / \mathrm{I}}$ reduction, most possibly for catalytic $\mathrm{CO}_{2}$ reduction ${ }^{42}$, as the position of this reduction wave is more negative than the standard reduction potential of $\mathrm{CO}_{2} / \mathrm{CO}$ $(-0.65 \mathrm{~V} \text { vs. } \mathrm{NHE})^{43}$. Moreover, the above results indicate that the catalysis driven by the photoexcited RuPS* should be thermodynamically accessible, as the driving force from the oxidative quenching pathway $(E=-0.84 \mathrm{~V} \text { vs. NHE })^{44}$ is more negative than the onset potential (ca. $-0.75 \mathrm{~V}$ ) of the catalytic wave.

According to above studies ${ }^{42,45}$, the catalytic mechanism of Co-MOF in $\mathrm{CO}_{2}$-to-CO conversion can be tentatively proposed, which was further verified by DFT calculation. A molecular prototype presenting the Co-complex moiety was subjected in computational studies (Fig. 5a). As illustrated by Fig. 5, the photocatalytic cycle begins with the photo-excitation of RuPS (Fig. 5b). Then, the exited RuPS* species can be oxidatively quenched by Co-MOL@GO catalyst to drive the $\mathrm{Co}^{\mathrm{II} / \mathrm{I}}$ reduction to form $\mathrm{Co}^{\mathrm{I}}$ species. The calculated potential for the reduction from $\mathrm{Co}^{\mathrm{II}}$ to $\mathrm{Co}^{\mathrm{I}}$ is $-1.04 \mathrm{~V}$ vs. NHE, approaching to the measured value (ca. $-0.94 \mathrm{~V}$ ) in the presence of $\mathrm{CO}_{2}$, further confirming the accessibility of this proposed mechanism. The $\mathrm{Co}^{\mathrm{I}}$ intermediate can react with $\mathrm{CO}_{2}$ to generate a $\mathrm{Co}-\mathrm{CO}_{2}$ adduct. Then a $1 \mathrm{e}^{-} / 1 \mathrm{H}^{+}$proton-coupled electron transfer takes place to generate a $\mathrm{Co}-\mathrm{COOH}$ species $(-1.09 \mathrm{~V})$. Finally, the $\mathrm{Co}-\mathrm{COOH}$ intermediate can release $\mathrm{CO}$ by cleaving the $\mathrm{C}-\mathrm{OH}$ bond to recover the $\mathrm{Co}^{\mathrm{II}}$ state. The remaining $\mathrm{RuPS}^{+}$species from the oxidative quenching pathway can be reduced to original RuPS by the TEOA, completing the photocatalytic cycle. During this photocatalytic process, the 2D GO not only serves as the template to reduce the surface energy of ultrathin nanosheets for constructing ultrathin MOLs with more exposed active sites, but also to supply conductive channels that can facilitate photoexcited electron transfer, which both play key roles in promoting the photocatalysis.

Notably, we have also operated DFT calculations on the alternate pathways for catalytic proton reduction to $\mathrm{H}_{2}$ and $\mathrm{CO}_{2}$ reduction to formate (Supplementary Fig. S21). It can be observed that the first protonation at the $\mathrm{Co}^{\mathrm{I}}$ intermediate is more thermodynamically unfavorable than the binding of $\mathrm{CO}_{2}(28.8 \mathrm{vs}$. $\left.10.7 \mathrm{kcal} \mathrm{mol}^{-1}\right)$, which should be the main reason for the selective production of $\mathrm{CO}$ over $\mathrm{H}_{2}$ and formate observed in the photocatalysis with Co-MOF-based catalysts.

\section{Discussion}

In summary, we have developed a facile and efficient strategy to construct ultrathin 2D MOLs with three metal coordination layers, where the MOLs are homogeneously distributed on the 2D GO template. The synergy between ultrathin MOL and the GO electronic conductor can greatly improve its intrinsic activity for photocatalytic $\mathrm{CO}_{2}$ reduction. The reduced $\mathrm{GO}$ can act as $2 \mathrm{D}$ template to support and stabilize the MOLs in a uniform thickness of ca. $1.5 \mathrm{~nm}$. Importantly, the GO can serve as efficient 
a

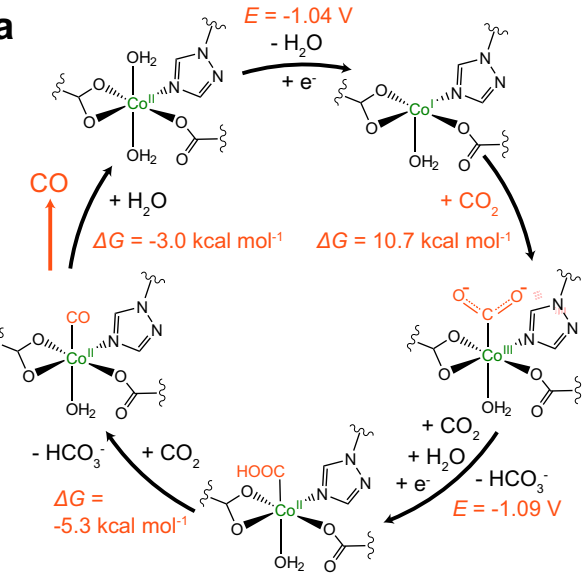

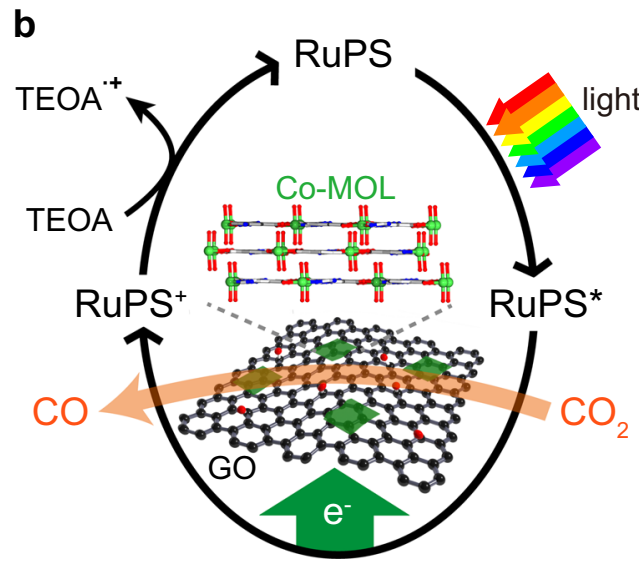

Fig. 5 Proposed mechanisms. a Calculated mechanism with the molecular unit of Co-MOF for photocatayltic $\mathrm{CO}_{2}$-to-CO conversion, showing the calculated redox potentials and free energy changes. b Proposed photocatalytic mechanism with Co-MOL@GO as the catalyst.

electron mediator to bridge the gap between heterogeneous catalysts and homogeneous antenna molecules, greatly contributing to the high activity. By merging these advantages, Co-MOL@GO exhibits excellent catalytic activity and high selectivity toward visible-light-driven $\mathrm{CO}_{2}$-to-CO conversion, achieving a recordhigh total CO yield of $3133 \mathrm{mmol} \mathrm{g}_{\mathrm{MOL}^{-1}}$ in $12 \mathrm{~h}$ among all the state-of-the-art MOF and MOL catalysts. This work paves an avenue on the economical preparation of ultrathin MOLs with advanced performance and demonstrates the key role of the electron mediator in dramatically promoting the photocatalysis.

\section{Methods}

Materials. All the chemicals, commercially available, were used without further purification. The used water was prepared by using a Milli-Q ultrapure water purification system. Electrodes and other accessories were all purchased from Gauss Union Technology Co., Ltd.

Instruments. PXRD data were collected by a Smart X-ray diffractometer (SmartLab 9 KW, Rigaku, Japan) with $\mathrm{Cu}$ Ka radiation $(\lambda=1.54178 \AA)$. EDS mapping images were acquired on an Environment Scanning Electron Microscope with a field emission gun (Quanta FEG 250, FEI, USA). The morphologies were recorded by a scanning electron microscope (Verios $460 \mathrm{~L}, \mathrm{FEI}, \mathrm{USA}$ ) and a transmission electron microscope (Talos F200 X, FEI, USA). The Co/Cd contents were determined by ICP-MS (iCAP RQ, Germany). Raman spectra were recorded on a highresolution laser confocal fiber Raman spectrometer (HORIBA EVOLVTION, HORIBA Jobin Yvon, France). More elemental information, especially for their valence states, was determined by XPS (ESCALAB250Xi, THERMO SCIENTIFIC, United Kingdom). The evolved $\mathrm{CO}$ and $\mathrm{H}_{2}$ were monitored by using an Agilent $7820 \mathrm{~A}$ gas chromatography with a thermal conductivity detector (TCD) and a TDX-01 packed column, the oven temperature was held constant at $60^{\circ} \mathrm{C}$, the inlet and detector temperature were set at 80 and $200{ }^{\circ} \mathrm{C}$, respectively. The gas analysis was also operated on another gas chromatography (GC-2014+ATF, 230 C, Shimadzu, Japan) equipped with two automated gas sampling valves, which contain a TCD and a flame ionization detector. The carrier gas used in the above two gas chromatography equipment was argon. The fluorescent spectra were conducted on a fluorescence spectrophotometer (F-7000, Hitachi, Japan). Time-resolved fluorescence measurements were measured with MicroTime 200 time-resolved confocal fluorescence instrument. The experimental data were analyzed by the SymPhoTime 64. Electrochemical measurements were operated on a CHI660D work station.

Synthesis of Co-MOF. In a 10-mL Teflon-lined autoclave, $\mathrm{CoCl}_{2} \cdot 6 \mathrm{H}_{2} \mathrm{O}(18.0 \mathrm{mg})$ and 5-(1H-1,2,4-triazol-1-yl)isophthalic acid $(9.0 \mathrm{mg})$ were dissolved in a mixed solvent containing $1.75 \mathrm{~mL}$ DMF, $0.5 \mathrm{~mL}$ water, and $0.25 \mathrm{~mL}$ acetic acid. After a 12 -h reaction under $130^{\circ} \mathrm{C}$, this mixture afforded violet crystals of Co-MOF, which were cleaned by water and ethanol, followed by drying at $60^{\circ} \mathrm{C}$.

Synthesis of Cd-MOF. In a 20-mL Teflon-lined autoclave, $\mathrm{Cd}\left(\mathrm{CH}_{3} \mathrm{COO}\right)_{2} \cdot 2 \mathrm{H}_{2} \mathrm{O}$ $(30.0 \mathrm{mg})$ and $5-(1 \mathrm{H}-1,2,4$-triazol-1-yl)isophthalic acid $(24.0 \mathrm{mg})$ were dissolved in a mixed solvent containing $2.0 \mathrm{~mL}$ water and $0.4 \mathrm{~mL}$ acetic acid. After a $24-\mathrm{h}$ reaction under $180^{\circ} \mathrm{C}$, this mixture afforded colorless crystals of Cd-MOF, which were cleaned by water and ethanol, followed by drying at $60^{\circ} \mathrm{C}$.
Synthesis of $\mathbf{Z n - M O F}$. In a $20-\mathrm{mL}$ Teflon-lined autoclave, $\mathrm{ZnCl}_{2}(30.0 \mathrm{mg})$ and 5 (1H-1,2,4-triazol-1-yl)isophthalic acid $(24.0 \mathrm{mg})$ were dissolved in a mixed solvent containing $2.0 \mathrm{~mL}$ water and $0.4 \mathrm{~mL}$ acetic acid. After a 24 -h reaction under $180^{\circ} \mathrm{C}$ this mixture afforded colorless crystals of $\mathrm{Zn}-\mathrm{MOF}$, which were cleaned by water and ethanol, followed by drying at $60^{\circ} \mathrm{C}$

Synthesis of Co-MOL@GO. The first step is the immobilization of $\mathrm{Co}^{2+}$ on the 2D GO nanosheets. The GO was synthesized according to previous report ${ }^{46,47}$. Then, $20 \mathrm{mg}$ GO and $0.5 \mathrm{~mL}$ water were added into $25 \mathrm{~mL}$ ethanol to obtain a homogeneous suspension after $1 \mathrm{~h}$ of ultrasonic treatment. Then, $0.1,0.3,0.5$, or $1.0 \mathrm{~mL}$ aqueous solution of $\mathrm{CoCl}_{2} \cdot 6 \mathrm{H}_{2} \mathrm{O}(0.1 \mathrm{M})$ was added into this $\mathrm{GO}$ suspension. After reaction at $80^{\circ} \mathrm{C}$ for $24 \mathrm{~h}$, the resulting Co@GO powder was isolated by centrifugation, followed by washing with water for several times and drying at $60{ }^{\circ} \mathrm{C}$. The second step is the growth of Co-MOL on the GO layers. Co@GO (20 $\mathrm{mg})$ and 5-(1H-1,2,4-triazol-1-yl)isophthalic acid $(10 \mathrm{mg})$ were added into a mixed solvent of $1.75 \mathrm{~mL}$ DMF, $0.5 \mathrm{~mL}$ water, and $0.25 \mathrm{~mL}$ acetic acid in a $10 \mathrm{~mL}$ Teflonlined autoclave. After reaction at $130^{\circ} \mathrm{C}$ for $4 \mathrm{~h}$, the Co-MOL@GO sample was obtained, which was washed by DMF, water, and ethanol, followed by drying at $60^{\circ} \mathrm{C}$. The mass contents of cobalt of Co@GO and Co-MOL@GO were analyzed by ICP-MS (Supplementary Table 2). The optimized catalyst was prepared with the addition of $0.5 \mathrm{~mL}$ aqueous solution of $\mathrm{CoCl}_{2} \cdot 6 \mathrm{H}_{2} \mathrm{O}(0.1 \mathrm{M})$ during synthesis.

Synthesis of Cd-MOL@GO and Zn-MOF@GO. First, 20 mg GO and 0.5 mL water were added into $25 \mathrm{~mL}$ ethanol to obtain a homogeneous suspension after $1 \mathrm{~h}$ of ultrasonic treatment. Then, $\mathrm{Cd}\left(\mathrm{CH}_{3} \mathrm{COO}\right)_{2} \cdot 2 \mathrm{H}_{2} \mathrm{O}(30.0 \mathrm{mg})$ was added into this GO suspension. After reaction at $80^{\circ} \mathrm{C}$ for $24 \mathrm{~h}$, the resulting Cd@GO powder was isolated by centrifugation, followed by washing with water for two times. Without drying, the as-prepared Cd@GO and 5-(1H-1,2,4-triazol-1-yl)isophthalic acid (24 mg) were added into a mixed solvent of $2.0 \mathrm{~mL}$ water and $0.4 \mathrm{~mL}$ acetic acid in a $20 \mathrm{~mL}$ Teflon-lined autoclave. After reaction at $180^{\circ} \mathrm{C}$ for $4 \mathrm{~h}$, the Co-MOL@GO sample was obtained, which was washed by water and ethanol, followed by drying at $60^{\circ} \mathrm{C}$. The mass contents of cadmium of Cd@GO $(18.1 \pm 0.5 \% \mathrm{Cd})$ and CdMOL@GO $(13.3 \pm 0.2 \%$ Cd) were analyzed by ICP-MS. Accordingly, ZnMOF@GO was intended to prepare by using $\mathrm{ZnCl}_{2}(30.0 \mathrm{mg})$ instead of Cd salt via systematically adjusting the synthesis conditions, while the PXRD results indicate the absence of $\mathrm{Zn}-\mathrm{MOF}$ crystal phase in the final sample.

X-ray crystallography. Single-crystal X-ray diffraction data were collected by an Xray single crystal diffractometer (XtaLAB Pro MM003Cu/Mo, Rigaku, Japan) equipped with $\mathrm{Cu} \mathrm{Ka}$ radiation $(\lambda=1.54178 \AA)$. The structures were resolved using the direct method and refined on $F^{2}$ by the full-matrix least-squares $\operatorname{method}^{48}$, which yields the positions of all non-hydrogen atoms and were all refined anisotropically. All hydrogen atoms of the ligand were placed in their calculated positions with fixed isotropic thermal parameters and included in the structure factor calculations in the final stage of refinement. The crystallographic data were supplied in Supplementary Table 1. The data with CCDC numbers 1965944, 2047069, and 2047070 for Co-MOF, Cd-MOF, and Zn-MOF contain the supplementary crystallographic information for this paper. The data can be obtained free of charge from The Cambridge Crystallographic Data Centre via http://www.ccdc.cam.ac.uk/

EIS measurements. EIS measurements were conducted on a CHI660D electrochemical station in a conventional three-electrode cell using a Pt plate as the counter electrode and an $\mathrm{Ag} / \mathrm{AgCl}$ electrode (saturated $\mathrm{KCl}$ ) as the reference electrode. The working electrode was a catalyst-loaded fluorine-doped tin oxide (FTO) glass slide. Prior to catalyst coating, the FTO slides were cleaned by 
sonication in ethanol for $30 \mathrm{~min}$ and dried at $353 \mathrm{~K}$. The boundary of the FTO glass was protected by using Scotch tape. Then, $2 \mathrm{mg}$ of catalyst was dispersed in $200 \mu \mathrm{L}$ of ethanol with $10 \mu \mathrm{L} 5 \%$ Nafion solution by sonication for $1 \mathrm{~h}$ to obtain a slurry, which was drop-cast onto the cleaned FTO glass. After drying overnight, the working electrode was further dried at $393 \mathrm{~K}$ for $5 \mathrm{~h}$ to improve the adhesion. EIS measurements were carried out at the open circuit potential. Prior to all measurements, the electrolyte $\left(0.2 \mathrm{M} \mathrm{Na}_{2} \mathrm{SO}_{4}\right.$ aqueous solution) was purged with $\mathrm{Ar}$.

Photocatalytic experiments. The photocatalytic reduction of $\mathrm{CO}_{2}$ to $\mathrm{CO}$ was conducted in a $17 \mathrm{~mL}$ home-made quartz reactor containing catalyst, $\left[\mathrm{Ru}(\text { phen })_{3}\right]$ $\left(\mathrm{PF}_{6}\right)_{2}$, TEOA, and $5 \mathrm{~mL} \mathrm{CH}{ }_{3} \mathrm{CN} / \mathrm{H}_{2} \mathrm{O}(v: v=4: 1)$ solution under $1 \mathrm{~atm} \mathrm{CO}_{2}$ atmosphere at $293 \pm 2 \mathrm{~K}$. After purging $\mathrm{CO}_{2}$ into this reaction system for $10 \mathrm{~min}$, the photocatalytic reaction was initiated by irradiation of a blue LED $(\lambda=450 \mathrm{~nm}$, light intensity $=100 \mathrm{~mW} \mathrm{~cm}$, irradiated area is approximate $0.5 \mathrm{~cm}^{2}$ ). A scalingup experiment was operated in a $67 \mathrm{~mL}$ home-made quartz reactor with the addition of $25 \mathrm{~mL} \mathrm{CH} \mathrm{CH}_{3} \mathrm{CN} \mathrm{H}_{2} \mathrm{O}\left(v: v=4: 1\right.$, irradiation area of $\left.2.0 \mathrm{~cm}^{2}\right)$ with identical concentrations of the above reagents. The generated gases in the headspace were analyzed by a gas chromatography, and the possible products in the solution were analyzed by an ion chromatograph. Each photocatalytic reaction was repeated three times to confirm the reliability of the data.

In situ TPV measurements. The working electrodes $(1 \times 2 \mathrm{~cm})$ were prepared by depositing samples $\left(150 \mu \mathrm{L} 5 \mathrm{mg} \mathrm{mL}^{-1}\right.$, dispersion liquid: $62.5 \%$ water, $25 \%$ isopropanol, and $12.5 \% 5 \mathrm{w} \%$ Nafion solution) on indium-tin oxide glass substrates. During the testing process, the working electrodes were kept dry or wet with $\mathrm{N}_{2}$ or $\mathrm{CO}_{2}$ saturated $\mathrm{CH}_{3} \mathrm{CN} / \mathrm{H}_{2} \mathrm{O}(v: v=4: 1)$, respectively. The samples were excited by a laser radiation pulse $(\lambda=355 \mathrm{~nm}$, pulse width $=5 \mathrm{~ns})$ from a third-harmonic Nd: YAG laser (Polaris II, New Wave Research, Inc.). The photocurrent is the ratio of the photovoltage to the internal resistance of the test systems.

Cyclic voltammetry. Cyclic voltammetric measurements were conducted on a CHI660D electrochemical station in a conventional three-electrode cell using a $\mathrm{Pt}$ wire as the counter electrode and another Pt wire as the pseudo reference electrode. Ferrocene $(\mathrm{Fc})$ was added and its reversible couple served as the internal potential

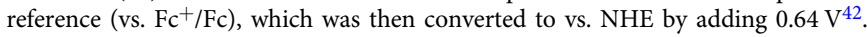
Catalyst-loaded glassy carbon disk electrode with $3 \mathrm{~mm}$ diameter was used as the working electrode. Prior to catalyst coating, the working electrode was polished by alumina oxide powder $(50 \mathrm{~nm})$ for $5 \mathrm{~min}$ and then cleaned by sonication in ethanol for $2 \mathrm{~min}$, followed by drying in air at room temperature. Then, $5 \mathrm{mg}$ of catalyst was dispersed in $4.95 \mathrm{~mL}$ of ethanol with $50 \mu \mathrm{L} 5 \mathrm{w} \%$ Nafion solution by sonication for $1 \mathrm{~h}$ to obtain a slurry. Then, $2 \mu \mathrm{L}$ of the prepared slurry was drop-cast onto the cleaned electrode and drying under ambient conditions for $2 \mathrm{~h}$. The supporting electrolyte is wet $\mathrm{CH}_{3} \mathrm{CN}$ containing tetra- $n$-butylammonium hexafluorophosphate $(0.1 \mathrm{M})$.

DFT calculation. All the calculations were performed with Gaussian 09 program ${ }^{49}$. All the structures were optimized at the B3P86 $6^{50,51} / \mathrm{def} 2 \mathrm{SVP}$ level of theory. Based on the crystal structure, some atoms were frozen during the optimizations. Frequency analysis calculations were performed to characterize the structures to be the minima. With B3P86/def2SVP optimized geometries, the energy results were refined by single-point calculations at the B3P86/def2TZVP level of theory. The solvation effect was also evaluated with the SMD solvent model at the B3P86/ def2TZVP level of theory. $\mathrm{H}_{2} \mathrm{O}$, in the presence of $\mathrm{CO}_{2}$, is chosen as the real proton source in $\mathrm{CH}_{3} \mathrm{CN} / \mathrm{H}_{2} \mathrm{O}$ system, as indicated by our previous reports ${ }^{45,52}$. The spin variation of Co centers has been considered and the thermodynamically stable species with specific spin states were adopted as the reasonable intermediates (Supplementary Table 4). For the redox potentials calculation, the result was calculated by Eq. (1),

$$
n E^{\Theta} F=\Delta G_{(\text {sol })}^{\Theta}
$$

where $\Delta G^{\Theta}$ (solv) is the free energy change of the reduction process at standard conditions, $n$ is the number of electrons, $F$ is the Faraday constant. According to the experimental $\mathrm{Fc}^{+/ 0}$ couple value of $-114.8 \mathrm{kcal} \mathrm{mol}^{-1}(-4.98 \mathrm{~V})^{53}$ in $\mathrm{CH}_{3} \mathrm{CN}$, the calculated redox potentials are referenced to $\mathrm{Fc}^{+/ 0}$ by subtracting $4.98 \mathrm{~V}$, then converting to vs. NHE by adding $0.64 \mathrm{~V}$. It should be noted that B3P86 should be the optimal functional by the consistency between the measured $\mathrm{Co}^{\mathrm{II} / \mathrm{I}}$ reduction potentials and the calculated values from a sequence of functionals in Supplementary Table 5 . We also note that the small basis set, def2SVP, is sufficient and efficient to yield results approaching to the experimental values $(-1.04 \mathrm{~V}$ vs. $-0.94 \mathrm{~V}$ ), as the full use of a larger basis set, def2TZVP, produced a similar value $(-1.02 \mathrm{~V})$ in parallel calculation while consuming a much longer calculation time.

\section{Data availability}

Supplementary figures and data are available from the authors. The data with CCDC numbers 1965944, 2047069, and 2047070 for Co-MOF, Cd-MOF, and Zn-MOF contain the supplementary crystallographic information for this paper. The data can be obtained free of charge from The Cambridge Crystallographic Data Centre via http://www.ccdc. cam.ac.uk/. Source data are provided with this paper.
Received: 9 July 2020; Accepted: 8 January 2021;

Published online: 05 February 2021

\section{References}

1. Peter, S. C. Reduction of $\mathrm{CO}_{2}$ to chemicals and fuels: a solution to global warming and energy crisis. ACS Energy Lett. 3, 1557-1561 (2018).

2. Zhang, $\mathrm{H}$. X. et al. Isolated square-planar copper center in boron imidazolate nanocages for photocatalytic reduction of $\mathrm{CO}_{2}$ to CO. Angew. Chem. Int. Ed. 58, 11752-11756 (2019).

3. Guo, Q. et al. Efficient and selective $\mathrm{CO}_{2}$ reduction integrated with organic synthesis by solar energy. Chem 5, 2605-2616 (2019).

4. Kuppler, R. J. et al. Potential applications of metal-organic frameworks. Coord. Chem. Rev. 253, 3042-3066 (2009).

5. Furukawa, H., Cordova, K. E., O'Keeffe, M. \& Yaghi, O. M. The chemistry and applications of metal-organic frameworks. Science 341, 1230444 (2013).

6. Muschi, M. et al. Formation of a single-crystal aluminum-based MOF nanowire with graphene oxide nanoscrolls as structure-directing agents. Angew. Chem. Int. Ed. 59, 10353-10358 (2020).

7. Alkhatib, I. I., Garlisi, C., Pagliaro, M., Al-Ali, K. \& Palmisano, G. Metalorganic frameworks for photocatalytic $\mathrm{CO}_{2}$ reduction under visible radiation: a review of strategies and applications. Catal. Today 340, 209-224 (2020).

8. $\mathrm{Li}, \mathrm{N}$. et al. Adenine components in biomimetic metal-organic frameworks for efficient $\mathrm{CO}_{2}$ photoconversion. Angew. Chem. Int. Ed. 58, 5226-5231 (2019).

9. Jing, X., He, C., Zhao, L. \& Duan, C. Photochemical properties of host-gues supramolecular systems with structurally confined metal-organic capsules. Acc. Chem. Res. 52, 100-109 (2019).

10. Wang, Y. et al. Hydroxide ligands cooperate with catalytic centers in metalorganic frameworks for efficient photocatalytic $\mathrm{CO}_{2}$ reduction. J. Am. Chem. Soc. 140, 38-41 (2018).

11. Kajiwara, T. et al. Photochemical reduction of low concentrations of $\mathrm{CO}_{2}$ in a porous coordination polymer with a ruthenium(II)-CO complex. Angew. Chem. Int. Ed. 55, 2697-2700 (2016).

12. Zhang, $H$. et al. Efficient visible-light-driven carbon dioxide reduction by a single-atom implanted metal-organic framework. Angew. Chem. Int. Ed. 55, 14310-14314 (2016)

13. Wang, S., Yao, W., Lin, J., Ding, Z. \& Wang, X. Cobalt imidazolate metalorganic frameworks photosplit $\mathrm{CO}_{2}$ under mild reaction conditions. Angew. Chem. Int. Ed. 53, 1034-1038 (2014).

14. Li, D., Kassymova, M., Cai, X., Zang, S.-Q. \& Jiang, H.-L. Photocatalytic $\mathrm{CO}_{2}$ reduction over metal-organic framework-based materials. Coord. Chem. Rev. 412, 213262 (2020)

15. Wang, D., Huang, R., Liu, W., Sun, D. \& Li, Z. Fe-based MOFs for photocatalytic $\mathrm{CO}_{2}$ reduction: Role of coordination unsaturated sites and dual excitation pathways. ACS Catal. 4, 4254-4260 (2014).

16. Wang, C., Xie, Z., deKrafft, K. E. \& Lin, W. Doping metal-organic frameworks for water oxidation, carbon dioxide reduction, and organic photocatalysis. $J$. Am. Chem. Soc. 133, 13445-13454 (2011).

17. Wu, L. Y. et al. Encapsulating perovskite quantum dots in iron-based metalorganic frameworks (MOFs) for efficient photocatalytic $\mathrm{CO}_{2}$ reduction. Angew. Chem. Int. Ed. 58, 9491-9495 (2019).

18. Kong, Z. C. et al. Core@shell CsPbBr $@$ @zeolitic imidazolate framework nanocomposite for efficient photocatalytic $\mathrm{CO}_{2}$ reduction. ACS Energy Lett. 3, 2656-2662 (2018).

19. Xiao, X., Zou, L., Pang, H. \& Xu, Q. Synthesis of micro/nanoscaled metalorganic frameworks and their direct electrochemical applications. Chem. Soc. Rev. 49, 301-331 (2020)

20. Lan, G. et al. Photosensitizing metal-organic layers for efficient sunlightdriven carbon dioxide reduction. J. Am. Chem. Soc. 140, 12369-12373 (2018).

21. Han, B. et al. Nickel metal-organic framework monolayers for photoreduction of diluted $\mathrm{CO}_{2}$ : metal-node-dependent activity and selectivity. Angew. Chem. Int. Ed. 57, 16811-16815 (2018).

22. Yang, L. et al. Two-dimensional metal-organic layers on carbon nanotubes to overcome conductivity constraint in electrocatalysis. ACS Appl. Mater. Interfaces 10, 36290-36296 (2018).

23. Zhu, W. et al. Selective reduction of $\mathrm{CO}_{2}$ by conductive MOF nanosheets as an efficient co-catalyst under visible light illumination. Appl. Catal. B-Environ. 238, 339-345 (2018).

24. Ye, L. et al. Assembly of highly efficient photocatalytic $\mathrm{CO}_{2}$ conversion systems with ultrathin two-dimensional metal-organic framework nanosheets. Appl. Catal. B-Environ. 227, 54-60 (2018).

25. Zhuang, L. et al. A surfactant-free and scalable general strategy for synthesizing ultrathin two-dimensional metal-organic framework nanosheets for the oxygen evolution reaction. Angew. Chem. Int. Ed. 58, 13565-13572 (2019).

26. Guo, Y. et al. Cooperative stabilization of the [pyridinium- $\mathrm{CO}_{2}-\mathrm{CO}$ ] adduct on a metal-organic layer enhances electrocatalytic $\mathrm{CO}_{2}$ reduction. J. Am. Chem. Soc. 141, 17875-17883 (2019). 
27. Lin, Z. et al. Metal-organic layers stabilize earth-abundant metal-terpyridine diradical complexes for catalytic C-H activation. Chem. Sci. 9, 143-151 (2018).

28. Shi, W. et al. Surface modification of two-dimensional metal-organic layers creates biomimetic catalytic microenvironments for selective oxidation. Angew. Chem. Int. Ed. 56, 9704-9709 (2017).

29. Li, F. L. et al. Large-scale, bottom-up synthesis of binary metal-organic framework nanosheets for efficient water oxidation. Angew. Chem. Int. Ed. 58, 7051-7056 (2019).

30. Han, J. et al. Reordering d orbital energies of single-site catalysts for $\mathrm{CO}_{2}$ electroreduction. Angew. Chem. Int. Ed. 58, 12711-12716 (2019).

31. Zhao, S. et al. Ultrathin metal-organic framework nanosheets for electrocatalytic oxygen evolution. Nat. Energy 1, 16184 (2016).

32. Huang, J. et al. Electrochemical exfoliation of pillared-layer metal-organic framework to boost the oxygen evolution reaction. Angew. Chem. Int. Ed. 57, 4632-4636 (2018).

33. $\mathrm{Wu}, \mathrm{H}$. et al. Mxene derived metal-organic frameworks. J. Am. Chem. Soc. 141, 20037-20042 (2019).

34. Ding, Y. et al. Controlled intercalation and chemical exfoliation of layered metal-organic frameworks using a chemically labile intercalating agent. J. Am. Chem. Soc. 139, 9136-9139 (2017).

35. Pan, Z., Zhang, G. \& Wang, X. Polymeric carbon nitride/reduced graphene oxide $/ \mathrm{Fe}_{2} \mathrm{O}_{3}$ : all-solid-state $\mathrm{Z}$-scheme system for photocatalytic overall water splitting. Angew. Chem. Int. Ed. 58, 7102-7106 (2019).

36. Zhou, D., Cui, Y., Xiao, P. W., Jiang, M. Y. \& Han, B. H. A general and scalable synthesis approach to porous graphene. Nat. Commun. 5, 4716 (2014).

37. Zheng, H. L. et al. Photochemical in situ exfoliation of metal-organic frameworks for enhanced visible-light-driven $\mathrm{CO}_{2}$ reduction. Angew. Chem. Int. Ed. 59, 23588-23592 (2020).

38. Niu, K. et al. A spongy nickel-organic $\mathrm{CO}_{2}$ reduction photocatalyst for nearly $100 \%$ selective CO production. Sci. Adv. 3, el700921 (2017).

39. Bonin, J., Robert, M. \& Routier, M. Selective and efficient photocatalytic $\mathrm{CO}_{2}$ reduction to CO using visible light and an iron-based homogeneous catalyst. J. Am. Chem. Soc. 136, 16768-16771 (2014).

40. Ouyang, T., Huang, H.-H., Wang, J.-W., Zhong, D.-C. \& Lu, T.-B. A dinuclear cobalt cryptate as a homogeneous photocatalyst for highly selective and efficient visible-light driven $\mathrm{CO}_{2}$ reduction to $\mathrm{CO}$ in $\mathrm{CH}_{3} \mathrm{CN} / \mathrm{H}_{2} \mathrm{O}$ solution. Angew. Chem. Int. Ed. 56, 738-743 (2017).

41. Chan, S. L., Lam, T. L., Yang, C., Yan, S. C. \& Cheng, N. M. A robust and efficient cobalt molecular catalyst for $\mathrm{CO}_{2}$ reduction. Chem. Commun. 51, 7799-7801 (2015).

42. Wang, J.-W. et al. Electrocatalytic and photocatalytic reduction of $\mathrm{CO}_{2}$ to $\mathrm{CO}$ by cobalt(II) tripodal complexes: low overpotentials, high efficiency and selectivity. ChemSusChem 11, 1025-1031 (2018).

43. Costentin, C., Drouet, S., Robert, M. \& Saveant, J. M. A local proton source enhances $\mathrm{CO}_{2}$ electroreduction to $\mathrm{CO}$ by a molecular Fe catalyst. Science 338, 90-94 (2012)

44. Ouyang, T. et al. Dinuclear metal synergistic catalysis boosts photochemical $\mathrm{CO}_{2}$-to-CO conversion. Angew. Chem. Int. Ed. 57, 16480-16485 (2018).

45. Wang, J.-W., Huang, H.-H., Sun, J.-K., Zhong, D.-C. \& Lu, T.-B. Syngas production with a highly-robust nickel(II) homogeneous electrocatalyst in a water-containing system. ACS Catal. 8, 7612-7620 (2018).

46. Hummers, W. S. \& Offeman, R. E. Preparation of graphitic oxide. J. Am. Chem. Soc. 80, 1339-1339 (1958).

47. Kovtyukhova, N. I. et al. Layer-by-layer assembly of ultrathin composite films from micron-sized graphite oxide sheets and polycations. Chem. Mater. 11, 771-778 (1999).

48. Sheldrick, G. M. Crystal structure refinement with shelxl. Acta Crystallogr. C. Struct. Chem. 71, 3-8 (2015).

49. Frisch, M. J. et al. Gaussian 16, Revision A.03 (Gaussian, Inc., 2016).
50. Perdew, J. P. Density-functional approximation for the correlation energy of the inhomogeneous electron gas. Phys. Rev. B 33, 8822-8824 (1986).

51. Becke, A. D. Density-functional exchange-energy approximation with correct asymptotic behavior. Phys. Rev. A Gen. Phys. 38, 3098-3100 (1988).

52. Wang, S. S. et al. Encapsulation of single iron sites in a metal-porphyrin framework for high-performance photocatalytic $\mathrm{CO}_{2}$ reduction. Inorg. Chem. 59, 6301-6307 (2020).

53. Sheng, M. et al. A nickel complex with a biscarbene pincer-type ligand shows high electrocatalytic reduction of $\mathrm{CO}_{2}$ over $\mathrm{H}_{2} \mathrm{O}$. Dalton. Trans. 44, 16247-16250 (2015).

\section{Acknowledgements}

This work was supported by National Natural Science Foundation of China (21722104, 21671032, 21971190, and 22071180), Natural Science Foundation of Tianjin City of China (18JCJQJC47700), China Postdoctoral Science Foundation (2020M683020), and the Fundamental Research Funds for the Central Universities (20lgpy87).

\section{Author contributions}

J.W.W and L.Z.Q contributed equally to this work. Z.-M.Z. conceived and designed this project, J.-W.W., L.-Z.Q., H.-D.N., Y.L., and M.L. performed the experiments, H.-H.H. carried out the DFT calculation, J.-W.W., S.Y., Z.-H.K., Z.-M.Z., Z.-H.K., and T.-B.L. analyzed the data, J.-W.W., Z.-H.K., and Z.-M.Z. wrote and revised the article. All authors participated in drafting the paper, and gave approval to the final version of the manuscript.

\section{Competing interests}

The authors declare no competing interests.

\section{Additional information}

Supplementary information The online version contains supplementary material available at https://doi.org/10.1038/s41467-021-21084-9.

Correspondence and requests for materials should be addressed to Z.-M.Z.

Peer review information Nature Communications thanks Rong Xu and other, anonymous, reviewers for their contributions to the peer review of this work. Peer review reports are available.

Reprints and permission information is available at http://www.nature.com/reprints

Publisher's note Springer Nature remains neutral with regard to jurisdictional claims in published maps and institutional affiliations.

Open Access This article is licensed under a Creative Commons Attribution 4.0 International License, which permits use, sharing, adaptation, distribution and reproduction in any medium or format, as long as you give appropriate credit to the original author(s) and the source, provide a link to the Creative Commons license, and indicate if changes were made. The images or other third party material in this article are included in the article's Creative Commons license, unless indicated otherwise in a credit line to the material. If material is not included in the article's Creative Commons license and your intended use is not permitted by statutory regulation or exceeds the permitted use, you will need to obtain permission directly from the copyright holder. To view a copy of this license, visit http://creativecommons.org/ licenses/by/4.0/.

(C) The Author(s) 2021 